raised; during convalescence, especially with the establishment of compensation in cardiac disease, the pulse often fails. Numerous observations seem to show that these differences in the reaction of the pulse do not depend on the amount of blood volume or the degree of maximal arterial pressure. It is not only conceivable but probable that signs of physiologic compensation which are absent during robust health or active disease, should become manifest when the normal vital energy is reduced or when, as a result of disease, the efforts of physiologic adjustment must become more strenuous to maintain the needful outflow and normal distribution of the blood mass. The older writers ${ }^{11}$ in this field were prone to attribute the pulsus paradoxus to mechanical interference with the normal blood flow, basing their position on the frequency with which the phenomenon attends pathologic adhesions between the mediastinal tissues. The facts that have been rehearsed seem to indicate that mechanical abnormalities within the chest may only indirectly cause pulse failure by exaggerating certain reflexes which under perfectly normal conditions are masked.

As already stated, failure of the elevated pulse does not seem to occur in persons showing signs of general arteriosclerosis. The thought may not be far amiss that this constant organic tendency of later life may in itself be a compensation which, by diminishing the distensibility of the vessels under the hydrostatic pressure of the blood, may more or less substitute that constant and necessary redistribution of the blood mass which has become impossible to a senile vasomotor apparatus.

\section{FINAL RESULTS OF CONSERVATIVE SUR- GERY OF THE TUBES AND OVARIES.}

ABRAM BROTHERS, B.S., M.D.

Adjunct Professor of Gynecology at the N. Y. Post-Graduate School and Hospital ; Visiting Gynecologist to Beth Israel Hospital and to the Yorkville Ilospital for Women and Chiloren. NEW YORK CITY.

It is an every-day experience in medical literature to read a surgeon's report of a series of "successful" operations. The surgeon expresses therein usually a feeling of satisfaction with the results of his work. Are the patients and family physicians equally content? What gratification or satisfaction is it to a woman to appreciate that she has escaped with her life, after a serious operation, only to discover that she continues to be an invalid or possibly a cripple? How many of these patients who have been "successfully" operated on pass on to the family physician, to the neurologist, or perhaps to the next surgeon for further attention? And how many of such patients would not rather have sent their blessings from the next world to their surgeon had he failed in noting a "successful" result and spared them the tortures of postoperative invalidism?

The older one grows in the practice of medicine and surgery the less is he apt to be carried away by the brilliancy of mere figures. The operator in surgery is, after all, only an artisan like the tailor or bricklayer with the additional equipment of a man educated in his special line. His specialty necessarily implies a thorough knowledge of anatomy and surgery as well as a cool head, steady nerve and an immense reserve stock of courage and backbone. If he does his work beautifully, skillfully and gracefully he becomes, in addition, the artist.

11. Cf., Hermann's Hdb. d. I'hysiologie, iv, 297.
But the man who combines the qualities of a good diag. nostician, careful thinker, artisan and artist is, after all, the best surgeon. Thus the average house surgeon to-day can point to a series of a dozen or twenty operations for appendicitis done neatly, quickly and, perhaps, without mortality. Yet he needs the experience of many mistakes in diagnosis as well as errors of judgment before he can be rated as a first-class surgeon.

From this rather pessimistic viewpoint have I tried to approach the subject in hand-namely, the true value of the conservative surgery of the adnexa. I have made little use of the literature of the subject and have tried to avoid being carried away by general impressions. Individual experiences extending over a period of many years have been the basis for the present study.

It is only proper to state at this point that the question of danger to life played a small rôle in this series of operations. In fact, in the hands of the average surgeon, in a properly equipped hospital, the mortality rate after this kind of work should be very low. In the collection of 160 cases of which I have records one patient died after operation and a second one is at present quite sick and in the hospital under the care of my successor to the service, Dr. S. W. Bandler. It is a good rule to study one's unsuccessful results in order to guard against the possibility of future error, and I shall describe these two cases at this point:

CAse 1 (Group 3).-History.-A young married woman, aged 28 , was referred to me by Dr. M. Cisin. On opening the abdomen a large tubo-ovarian abseess was discovered in the left side. It was ruptured during the subsequent enucleation and removal. The right tube was the seat of a purulent sal. pingitis and was amputated at the horn of the uterus. The ovary on this side was normal, and, in order to give the patient a chance to overcome the existing sterility as well as to retain the menstrual function, it was attached to the right uterine cornu. The broad ligament gap was brought together on each side with sutures in such a manner as to keep the uterus from falling backward. The extensive adhesions to the omentum and intestines were carefully separated and the diseased appendix was removed. Because of the escape of pus from the left tube ruptured during the operation the wound was drained. At the close of the operation the pelvis was clean and dry. The same evening the patient passed into a state of collapse which was attributed to postoperative shock. Under stimulating treatment she rallied. The wound being drained and little blood having appeared in the dressing, we were entirely misled in estimating the true condition of affairs. Several days passed before the secondary hemorrhage which had taken place was recognized, and, as the patient had emerged from the shock and her pulse was of fair quality, there was no indication for a secondary operation at this time. The wound was opened sufficiently to allow of the free escape of the blood accumulation. In the course of weeks, this case reduced itself to a purulent discharge through the abdomen and the patient passed through an attack of septic pleuropneumonia. After a valiant battle for life she finally succumbed to chronic sepsis and slow exhaustion at the end of ten weeks.

CASE 2 (Group 3).-History.-A young married woman, 31 years old, sterile. After preliminary eurcttage and drainage of the uterus, an abdominal section was made. The omentum, densely adherent to the anterior parietal peritoneum, was carefully detached. The right ovary was buried in firm adhesions so that during delivery it was severely mutilated and torn into two fragments. The right Fallopian tube was probed and its canal proved to be completely obliterated; it was therefore amputated at the horn of the uterus on this side. The half of the ovary which was still in the depths of the pelvis was sutured so as to check bleeding. The detached half was scwed to the horn of the uterus. On the left side the diseased obliterated tube was amputated at the uterine cornu. No attemnt was made to tear this ovary ont of its lodgings. The appendix was removed and the wound was drained. $A$ con 
dition of suppuration developed for which it was decided to make a vaginai counter-opening. Through an oversight in preparation the bladder was not emptied according to routine, and, in making a counter opening through the lateral vaginal fornix, the bladder was inadvertently injured; so that the patient, besides her abdominal sinus, is now the unfortunate possessor of a urinary fistula which will probably require another operation for its cure.

With the exccption of the two cases above narrated, all of the others were discharged from the hospital as "cured." Of these 158 cases I regret to say that I have been able to examine and to secure subsequent reports from only 85, the others having passed out of my horizon, so that nearly one-half of my work in conservative surgery of the adnexa is at present beyond my reach.

'The history of conservative surgery of the pelvis dates back a long tine. Thus the evacuation of pelvic abscesses, or adherent pus tubes, per vaginam or rectum or through the abdominal wall, was practiced by surgeons in the early days. The curved, sharp-pointed bistoury having been plunged into the abscess, the blade was washed, or possibly only wiped dry, and returned to the trousers pocket. As for pelvic hematocele, which we now know to be regularly the result of ectopic gestation, we learn that Ruyschk of Amsterdam alludes to it as far back as the year 1\%3\%, and that in 1831 Recamier opened into one under the impression that he was opening a pelvic abscess. How comparatively seldom to-day do we treat pelvic collections of blood or pus by simple incision and dranage? And yet conservative surgery of this kind still has its appropriate place.

THE BEGINNING OF ASEPTIC SORGERY.

Then followed the period in which tubes and ovaries were needlessly sacrificed because surgeons found that the introduction of asepsis reduced the mortality of intraperitoneal operations. With what degree of pardonable pride did not the surgeons of twenty years ago allude to their "successful" work in this direction? It was the neurologist and the general practitioner who called a halt to this form of malpractice. Dr. A. Jacobi was at that time quoted as having said that he preferred to see most of these ovaries in the abdominal cavities of women than in the surgeon's jar. The result of this reaction was sudden and decisive, so that to-day it is, indeed, rare to meet a surgeon who is willing to sacrifice healthy or micro-cystic ovaries for the cure of dysmenorrhea. hysteria and epilepsy.

If the gynecologist deserves to have a place in surgery at all-and this scems to be denied him by certain large hospitals and by some of the best general surgeons - it is because of much of the work done in this particular branch of pelvic surgery. It is not the purely mechanical part of his work which justifies the gynecologist to pose as a specialist. It will be conceded that every good surgeon should be able to do this kind of work equally well provided his opportunities for gaining experience occur often enough. It is in the laboratory, in the clinic and in the operating room that the student devoted to diseases peculiar to women gets his advantage over the general practitioner of medicine and over the general surgeon. The result is a clearer insight into diagnosis, a lietter judgment as to the proper course to pursue, and greater familiarity and facility in the technical details of gynecologic operations.

About ten or twelre years ago leading gynecologists conceived the idea of substituting conservative surgery of the adnexa for the radical work then in vogue, the object being not to reduce the mortality rate but to re- duce the morbidity aftrr operations on the tubes and ovaries. One of the bus of these early papers was written by Pozzi, and in it he describes the technic of conservative surgery of the ovaries-a technic practically adopted by all operators to-day. The conservative surgery of the tubes owes its chief impetus to American gynecologists-chiefly to the writings and work of the late Dr. A. P. Dudley. In developing this kind of work operators banished the old mass ligature, which, by compressing nerves in the constricted tissues, was responsible for much of the postoperative suffering so often met with under the older methods of dealing with pedicles. Silk ligatures, which used to be followed frequently by sinuses of the abdominat watl. were similarly discarded, so that most operators now use catgut excluisively.

THE OVARY.

The ovary presents a large field for conservative work when it is the seat of cystic disease. The microcystic ovary, as pointed out by Welch of Baltimore and $\mathrm{W}$. Gill Wylie of New York, is not in itself a pathologic lesion of sufficient importance to justify the opening of the abdomen. If the abdomen is opened, however, for other reasons and such ovaries are discovered the cysts may be punctured with a needle or, as the German operators prefer, with the point of an actual cautery (ignipuncture) so as to destroy thoroughly the cyst wall. As a rule, all of the cysts will not be reached by e:ther method, but, as the lesion is ordinarily insignificant, this need cause little regret. Another method to attack such ovaries, particularly when there is reason to feel that they are the seat of abnormal internal tension, is to split the cortex or bisect the ovary and prick the little cysts seen in each segment. That tension in an ovary is relieved after bisection there can be no doubt, but often it will be necessary to suture the two halves together again in order to control oozing of blood, which, to my mind, nullifies the advantages of the operation. For this reason in recent years $I$ have at times reduced the size of swollen micro-cystic ovaries by exsecting a longitudinal segment, like a slice from a watermelon, and then have stitched the wounded surfaces together.

The large unilocular or multilocular ovarian cysts, reaching to the size of a cocoanut, football or larger, were formerly subjected to complete removal. To-day, thanks to the labors of Boldt and some German operators, it has been proven that enough ovarian tissues can be found at the base of these tissues to leave a functionating ovary of greater or lesser size. The tumor is circumcised and cut out at its base, where usually some healthy-looking ovarian tissue will be recognized, which can be left. No ligation of vessels is necessary. The remnant of ovarian tissue is now sewed in such a manner that its raw surfaces are brought in close apposition. I have known large tumors of both ovaries to be treated in this manner in which menstruation subsequently followed its normal course.

The smaller ovarian cysts, ranging in size from a hazelnut to a plum, are either developments from a Graafian vesicle or a corpus luteum. When their contents are hemorrhagic they are known as hematomata. Ordinarily they can be exsected with the sac intact, or the tumors can be incised and the sac wall drawn out with forceps or scraped off with the knife. In either case a few stitches readily obliterate the bed of the cyst. Some operators like to turn in the peritoneal coat of the ovarian wound with Lembert sutures, but I regard this as an unnecessary refinement. 
Ovaries which have undergone fibroid degeneration are seldom amenable to conservative surgery. At least I have never met any which could be thus treated. Malignant disease of the ovaries contraindicates any attempt at conservatism. Ovaries in juxtaposition with pus tubes or purulent pelvic collections can safely be left undisturbed or may be subjected to partial exsection. These ovaries, if not the seat of the abscess at the time of operation, seldom, in my experience, give rise to trouble later. If they are involved in a suppurative process and form part of a tubo-ovarian abscess they should ordinarily be removed. Exceptionally they may be attacked through the vaginal route. On a number of occasions I was able to incise and drain ovarian abscesses through a vaginal incision. In such cases it is likely that a certain amount of healthy ovarian tissue was left.

THE FALLOPIAN TUBES.

The desirability of applying conservative surgery to pus tubes is open to question in my mind. I know that, per vaginam, $I$ have frequently left such tubes after incision and packing. I have followed one case for twenty years in a woman who subsequently was never sick and gave birth to a number of children. Kelly and Boldt, who make use of this plan in very young women, concede that such cases may require secondary operations from time to time. Hence, where I have my choice, my practice is in favor of total and complete ablation of pus tubes down to the horn of the uterus. Experience with secondary operations for infected stumps has driven me to this position.

Although it is possible to save tubes and cure hematomata by pelvic incision and drainage, I seldom make the effort to attack cases of ectopic gestation, which is the usual underlying condition in these cases, excepting by laparotomy and the removal of the pregnant tube.

When, however, the Fallopian tube is twisted or elbowed as a result of pelvo-peritonitis it can be restored to its shape by simply breaking it away from its adhesions. When the outer half or less of the tube is the seat of hydrosalpinx it can be remored, leaving the inner portion to be attached to the ovary. I have never seen an ectopic pregnancy to follow such attachments, although this has been one of the objections to the procedure. Intra-ligamentary crsts can often be safely attacked and cured by vaginal incision and drainage, although it is best to remove them intact from above.

In do:ng plastic work on the tubes and ovaries the tube is amputated in what appears to be healthy structure at the proximal attachment of the hydrosalpinx (or perhaps otherwise diseased and obstructed tube). A sound, probe or wire is passed along the lumen of the tubal stump until it enters the uterine cavity. If this prores to be obliterated there is no object in further attempts at plastic work and it is well to amputate the stump down to the horn of the uterus. Should a canal bo demonstrable the open end of the tubal stump is split toward the uterus a distance of about a half inch and the mucous lining is sewed to the peritoneal coat with a half dozen fine catgut stitches. The ovary, or a healthy portion of it, after having received proper surgical attention, is attached by its smooth, uninjured surface to the peritoneal surface of the tube at the newly made ostium. If these precautions are not taken there is reason to believe that the whole work is in vain and that the new opening made is subjected to permanent occlusion. The plastic work, properly done, gives the patient a new ehance for overcoming an otherwise hopeless barrier to impregnation.
Exsection of tubes or portions of tubes for the purpose of inducing sterility is not, properly speaking, to be regarded as conservative surgery. There are, however, clear indications for its performance and I have had occasion to resort to the procedure a number of times. A segment of each tube is caught between two ligatures and exsected. It has rarely happened after this was done that a new connection formed to permit of the union of an ovum with a spermatozoon-never in my own cases.

\section{THE PELIIC PERITONEUM.}

Finally we meet with cases in which, with or without lesions of the tubes and ovaries, the chief pathologic changes have involved the pelvic peritoneum. These changes are ordinarily dependent on the puerperium or occur after abortions, gonorrhea or tuberculosis. The management of pelvic adhesions is often very unsatisfactory. Under early postoperative mobilization as advised by Ries, Boldt and myself, I believe that reformation of such adhesion is prevented. Under most conditions they can be separated or broken up in a fairly satisfactory manner. In certain cases there is considerable risk of injuring the intestines, tubes or ovaries while digging the adnexa or uterus out of dense adhesions. Where the ovaries or tubes run the risk of great damage during delivery it is wiser to proceed with their complete extirpation, as my experience leads me to believe that the retention of such mutilated or damaged adnexa only too often threatens the ultimate comfort and health of the patient and leads to secondary operations for their final removal.

In quite a few cases, however, it is possible to release the adnexa from surrounding adhesions and to preserve them in whole or in part for purposes of plastic work. Where adhesions are visible it is well to work with the aid of the sight and fingers, using ligatures when necessary, particularly when dealing with the omentum. When the integrity of the intestinal wall is threatened I prefer to leave bits of tissue, like the wall of a crit or abscess sac, as I regard an injured intestine as nue of the most serious complications of an operation. In many cases, however, it is possible to release more or less completely the adnexa from adhesions and to preserve them intact without subjecting them to enough damage to demand their removal. In the ease of arthesions deeply situated in the pelvis a line of cleavage will usually be discovered by the experienced finger and the adnexa brought up for inspection. One must work often by the sense of touch, and I advise s'ow, gentle manipulation with the palmar surface of one or two fingers.

Total extirpation of both tubes and ovaries implies three things: 1 , Cessation of menstruation; 2. hopele'ss sterility; 3 , nervous breakdown from the sudklen onset of the menopause. Whether or not one is inclined to accept the views of the French authorities who claim that the ovaries secrete a juice which is necessary for the normal state of the female economy and the sudden removal of which tends to disturb the balance of the simpathetic nervous system and to produce various disturbances, the fact remains that in the normal climac. teric the menopause is reached in $a^{*}$ slower and much more gradual fashion. The system seems to be prepared, in the normal course of events, for a cessation of menstruation by the gradual and physiologic atrophy of the ovaries. But in the artificial menopause induced after operation the sto- bursts suddenly and the entire nervous system is shocked with its violence. 
Hence conservative surgery of the adnexa has for its main objects: 1 , The remoral of diseased tissues; 2 , the relief of various forms of disturbances and suffering; 3 , the preservation of menstrual function; 4, the possible overcoming of pathologic conditions which are responsible for sterility; 5 , the avoidance of the collapse symptoms resulting from the sudden onset of the menopause; 6 , the prevention of senile atrophy and changes which take place about the vulva and vagina and shorten the enjoyment of sexual life in many cases.

\section{a comparison of conservative and radical cases.}

Over conservative methods, radical adnexal surgery has the advantage of positively removing all of the pathologic tissues (and frequently much more than necessary), giving the patient on the whole a better guarantee against subsequent operations. It does not guarantee immunity from postoperative pain because adhesions are still possible and nerve inclusions can not always be avoided. Those who remove the uterus regularly with the diseased adnexa also submit their patients to a greater operative risk, and I am eatisfied that this organ is only too often thus unnecessarily sacrificed.

Conservative surgery, on the other hand, aims at saving healthy tubes or ovaries in whole or in part and never sacrifices the uterus. Besides removing the tissues which are distinctly diseased, it aims at preserving the menstrual function. It occasionally overcomes the obstacles to impregnation. It always inhibits the local and general results of the sudden menopause.

For the purposes of better study I have divided my work in this field arbitrarily into three groups of cases: Group 1, in which one tube or a portion of one tube was removed; and also incision and drainage of a tube. Group 2, in which ovaries were bisected or punctured and cysts exsected; also incision and drainage of an ovary. Group 3, in which adhesions were broken up with or without plastic conservative surgery of the tubes or ovaries. The three groups comprise 160 cases, of which 26 belong to the first class, 44 to the second, and 90 to the third class.

To judge properly postoperative morbidity one must be in possession of plenty of facts. Hence only multiplied experiences of numerous operations can determine percentages with any degree of fairness. The operator who sees his patient subsequent to operations will adjudge the result as satisfactory if he finds the pelvic organs in good condition and functionating, the abdominal scar firm, and the general nutrition of the patient good. The family physician, however, listening to postoperative tales of distress dependent on nervous or gastrointestinal derangement and exerting every endeavor to relieve the patient of a persistent dysmenorrhea or dyspareunia, will be apt to take a more pessi"mistic view of the "successful" operation.

Looking at my work from my own viewpoint and asking the thoughtful reader to make full allowance for the "personal equation" inevitably present. I beg to submit the following observations on postoperat ve morbidity.

In Group 1 (conservative work in 26 patients on the Fallopian tubes) I have noted unsatisfactory results in two cases. One of these patients a year later was suffering from a pelvic inflammatory mass which could be distinetly felt. In the second case the patient required a secondary laparotomy for the removal of an infected stump left after the first operation. One of the patients in this series of cases died suddenly of a cancer of the rectum which does not reflect on the propriety of the operation previously done by me.
In Group 2 (conservative work on 44 patients on the ovaries) six patients had good reason to complain of postoperative suffering. A distinct pelvic mass was present in one case and a secondary operation was rcquired in another.

In Group 3 (conservative work in 90 patients with adhesions of tubo-ovarian disease) I have notes of seven unsatisfactory results. In three cases of this group the patients presented pelvic inflammatory masses, subsequent to operation, which caused much suffering. Four patients required secondary operations. The two cases alluded to in the early part of this paper belong to this group.

Although the total number of secondary operations done in my cases after conservative surgery amounts to only six cases, $I$ readily concede that this figure is contestable on the ground that I lost sight of a certain number of my patients after they left the hospital. Some of these patients possibly required secondary operations elsewhere at some subsequent period. As six patients required secondary operations in the cases which I was able to follow it may be fairly assumed that six others were submitted to similar operations in the other half lost from view. Thus I infer that the maximum neceseity for secondary operations after conservative adnexal surgery does not exceed 7 per cent.

\section{WHAT IT MEANS.}

Too much stress can not be laid on the importance of reaching some conclusions in this matter, for it is in regard to the guarantee against secondary laparotomy that radical adnexal surgery has a distinct advantage over conservative work. If investigations by other operators, based on careful and positive observations, should prove that the morbidity after conservative adnexal surgery is so great as to require an unwarranted number of secondary operations then the advantages of preserving ovulation, menstruation and possible fecundity are clearly outbalanced by the inconvenience, if not actual danger to life, of a secondary laparotomy. The results which my own experiences thus far have reached, however, point the other way.

The general health of patients was noted in eighty-five cases at periods of time varying between one month and twelve years after operation. Of these thirty-five women were seen within six months from the time of operating, and in these the postoperative morbidity reached the highest percentage. While it is possible that many of these women seen within this period of time ultimately went on to perfect recovery, it is just as logical to assume that others remained more or less permanent invalids and had to submit to further operative intervention. Taking all in all, I estimate the postoperative morbidity after conservative surgery of the arlnexa to be about 23.5 per cent. In other words, I believe that three out of four of these women are cured or left free from important symptoms attributable to the pelvic structures.

That many women who keep complaining for the first six months or year after operation gradually pass into an improved condition or perhaps become so accustomed to their ailments that they cease to complain is a frequent experience in the domain of surgery. Of fifty cases examined by me after the lapse of six months from the time of the conservative operation only 18 per cent. were regarded as ill enough to require treatment. We must, therefore. never hastily advise secondary operations. I have numerous notes in my history books of 
"swollen ovaries" or "pelvic exudates" following operations which disappeared entirely in the course of time.

To attempt to specify the exact value of conservative adnexal surgery in relieving preoperative symptoms is a very difficult task. For after all many of these symp- * toms are purely subjective or relative. What may be described as a burning sensation in the pelvis by one woman will be regarded as intolerable torture by another. Thus the amount of pain which the individual woman actually suffers will often be in direct proportion to the development of her nervous system or imagination. Similarly, suffering ascribed to dysmenorrhea, menurrhagia or dyspareunia will be carefully estimated by thu discriminating medical attendant with these possibilities in view.

Postoperative menstrual disturbances were noted fourteen times in sixty-six cases. They consisted of metrorrhagia, amenorrhea of different grades, and drsmenorrhea. They occurred in Group 1 twice, in Group 2 three times in Group 3 nine times. Whether 21 percent. represents the exact truth in regard to the estimate of postoperative menstrual disturbances I leave to other investigators to determine.

The most difficult symptom to estimate after a pelvic operation in women is pain. I have reached that stage in which $I$ listen to the paticnt's talk of pain and try to determine approximately its value by judging how much of a disturbing factor it is on sleep, on appetite, on general comfort and in interfering with nutrition. Hence it is that complaints of pain after operation I frequently take cum grano salis; and this may account for the fact that in 80 per cent. of my postoperative observations I regarded the pains as being insufficient to reflect on the value of the original operation.

\section{TIIE CLliE OF STERILITY.}

When in the freshness of virgin enthusiasm I took up plastic adnexal surgery I had before my mind's eye the brilliant results of $\mathbb{W} . \mathbf{K}$. Polk and others in overcoming that hopeless form of sterility resulting from lesions of the tubes, ovaries and pelvic peritoneum. I felt that there was logic in the statement that the lumen of a distorted Fallopian tube conld be restored to its normal caliber and that portions of healthy ovary could be attached to stumps of repaired tubes so that the impregnated ovum would have a fair chance of migrating via the newly-created channel into the uterus for lodgment and further development. It is, therefore, with a certain feeling of disappointment that I find notes of only twelve cases-or 7.5 per cent.-of subsequent pregnancies. I think that this represents more clearly the truth than any of my other postoperative observations in these cases. As I have bcen peculiarly attracted to the study of every phase of sterility in women and its management I have regularly asked my patients after operation to report in the event of subsequent impregnation and childbirth. Still it will be conceded that evon this small percentage of successes is a triumph of which modern surgery has good reason to be proud.

Is conservative surgery of the adnexa only a passing craze or fashion to be wiped out of existence by newer medical or surgical methods? Is it open to better technic and improvement? $\mathrm{Or}$ is it to drop out of sight as an experiment which has not stood the test of time only to yield to its older rival-the complete reIf moval of diseased adnexa? I think not. It seems to me that with a mortality rate which, in good hands, should be very low and with a morbidity rate (requiring occasionally a secondary operation) of one in four, the methods of conservative surgery of the adnexa have come to stay.

112 East Sixty-first Street.

\section{REPORT OF ONE HUNDRED AND SEVEN CASES OF CANCER TREATED WITH THE ROENTGEN RAY, WITH COMMENTS THEREON.*}

\author{
ENNION G. WILLIAMS, M.D. \\ RICHMOND, VA.
}

Although the number of cases of cancer which I report is not large, yet they represent the work as it has turned up in my Roentgen ray practice. To appreciate properly the value of the $x$-ray treatment one must realize the vast difference between different cases of cancer from a clinical standpoint. Microscopically they are all more or less the same in structure, but clinically they vary from the almost inactive superficial sore, a slight degree removed from a senile keratosis, to a large growth associated with a general carcinosis.

\section{TIIE NEED OF DISCRIMINATION.}

By laymen, and occasionally even by physicians, all cancers are placed in the same class of malignancy. If the $x$-ray fails to heal one case of cancer their whole faith in the cficicacy of that treatment of cancer is wealened; on the other hand, there are those who hearing of the $x$-ray healing one case will jump to the conclusion that all cases should be healed by the ray. It is only possible, therefore, to approach an estimate of the value of the ray by having a large number of cascs and dividing them into classes which are more or less similar and susceptible of comparison.

By the study of the results of the treatment of a large number of cases so classified a fairly accurate estimate of the value of the ray can be determined. Should the surgeons furnish us with a similar classification of cases, treated by their methods together with the results, the value of the ray could then be determined as compared with surgical methods.

I have divided the cases into six classes, according to the extent of the involvement. Many senile keratoses were healed with the ray, but they were not considered carcinomatous and hence are not included in this classification. Senile keratoses should be looked on with suspicion and considered as pre-cancerous conditions. They not infrequently develop into well-defined squamouscelled carcinomas. In the same class of pre-cancerous conditions are moles which occasionally become irritated. These conditions when ulcerated heal quickly under the influence of the Roentgen ray. The unirritated mole does not yield so readily.

I have not used the term epithelioma bccause all carcinomas are tumors of epithelial cells, and only the loose use of the term has by custom restricted its meaning to that of a squamous-celled carcinoma, or epidermoid carcinoma, or, as it is commonly called, a skin cancer.

Growths resulting from keratoses are usually, if not always, squamous-celled carcinomas. Some growths resulting from moles are squamous-celled carcinomas and some are composed of endothelial cells. Such endo-

* Read bofore the American Roentgen Ray Society, Oct. 3, 1907, Cincinnat, Ohio. 